\title{
Exploring consumer intake, knowledge and perceptions of food supplements containing botanicals and their promotion on social media platforms: the \#SupplementsOnSocials survey
}

\author{
B. McDaid ${ }^{1}$, N. O'Kane ${ }^{1,2}$, J.V. Woodside ${ }^{1,2}$, C.E. Neville ${ }^{2}$ and A.P. Nugent ${ }^{1,3}$ \\ ${ }^{1}$ Institute for Global Food Security, Faculty of Medicine Health and Life Sciences, Queen's University Belfast, Belfast, \\ $U K$, \\ ${ }^{2}$ Centre for Public Health, Faculty of Medicine Health and Life Sciences, Queen's University Belfast, Belfast, UK and \\ ${ }^{3}$ Institute of Food and Health, School of Agriculture and Food Science, University College Dublin, Dublin, Ireland
}

Botanicals are found in some food supplements (FS) and, while little is known about their intake, botanical food supplements (BFS) appear to be widely marketed ${ }^{(1)}$. Social media (SM) is a popular form of advertising, with the UK SM advertising spend in 2019 being approximately $£ 3.6 \mathrm{bn}^{(2)}$. In popular culture, the topic of FS advertising on SM platforms is discussed, particularly regarding the sources and content of the advertisements ${ }^{(3)}$, but this has not been examined rigorously. This research explored self-reported intakes of FS and BFS by social media users, and evaluated consumer knowledge, attitudes, and perceptions of SM advertising of FS and BFS to inform a subsequent source and content analysis of SM posts promoting, selling or discussing BFS.

An online survey was developed to investigate self-reported use of FS/BFS and SM, and consumer knowledge and perceptions of SM advertising of FS/BFS. The survey was hosted on Qualtrics and data were collected over a 3-week period. Participants $(\mathrm{n}=213)$ were recruited via SM (Twitter, Facebook, Instagram, LinkedIn) and were 18+ years of age. Data were quality controlled and analysed in SPSS using descriptive statistics, while free text questions on consumer perceptions were analysed thematically. Ethical approval was granted by the Faculty of Medicine, Health and Life Sciences at Queen's University, Belfast.

Respondents were $86 \%$ Female, with 61\% aged 18-34y. A high number (93\%) reported either current or past use of FS. The most common reasons for use of FS by current users $(n=117)$ were 'overall health' $(41 \%)$, 'immunity purposes' $(20 \%)$, and 'to fulfil part of their dietary requirements' $(12 \%)$. Similar patterns were identified among past users. Vitamin D was the most frequently used FS (21\%), followed by multivitamins (15\%), and vitamin C (14\%). BFS intake was reported by $<6 \%$ of the sample. While $75 \%$ of respondents reported observing advertisements for FS on SM platforms, only $43 \%$ had heard of the term 'botanical food supplement'. However, thematic analysis of open-ended questions showed that respondents believed BFS to be either 'plant-based' substances, 'natural' substances, 'herbal' substances, or a combination of these three terms. Furthermore, 24\% of respondents $(\mathrm{n}=51)$ were able to provide named examples of influencers/celebrities who they had observed advertising FS/BFS products on SM.

Overall, self-reported intake of BFS by SM users was low and consumers claimed unfamiliarity with the term, however, most appeared to have an overall understanding of what a BFS constitutes. Consumers are being exposed to FS advertising on SM and are aware of individuals and brands advertising in this manner. An analysis of SM data informed by the results of the survey will provide further understanding about what specific SM advertisements are present on SM platforms and the individuals or organisations who are promoting them.

\section{References}

1. Lee et al. (2015) BMC Nutr 1, 43

2. Statista (2020) Social media advertising spending in the UK from 2011-2019.

3. Mospan CM \& Alexander KM (2018) Curr Pharm Teach Learn 10(11), 1507-1511. 